\title{
Synthesis of phosphonate-functionalized polystyrene and poly(methyl methacrylate) particles and their kinetic behavior in miniemulsion polymerization
}

\author{
Anke Ziegler • Katharina Landfester • \\ Anna Musyanovych
}

Received: 12 March 2009 /Revised: 20 July 2009 / Accepted: 22 July 2009 / Published online: 11 August 2009

(C) The Author(s) 2009. This article is published with open access at Springerlink.com

\begin{abstract}
Phosphonate-functionalized polymer nanoparticles were synthesized by free-radical copolymerization of vinylphosphonic acid (VPA) with styrene or methyl methacrylate (MMA) using the miniemulsion technique. The influence of different parameters such as monomer and surfactant type, amount of vinylphosphonic acid on the average particle size, and size distribution was studied using dynamic light scattering and transmission electron microscopy. Depending on the amount and type of the surfactant used (ionic or non-ionic), phosphonatefunctionalized particles in a size range from 102 to $312 \mathrm{~nm}$ can be obtained. The density of the phosphonate groups on the particle surface was higher in the case of using MMA as a basis monomer than polystyrene. The kinetic behavior of VPA copolymerization with styrene or MMA using a hydrophobic initiator was investigated by reaction calorimetry. Different kinetic curves were observed for miniemulsion (co)polymerization of styrene- and MMA-based nanoparticles indicating different nucleation mechanisms.
\end{abstract}

Keywords Miniemulsion · Radical copolymerization . Functionalized nanoparticles · Phosphonate groups · Calorimetric measurements

\footnotetext{
A. Ziegler $\cdot$ K. Landfester $\cdot$ A. Musyanovych $(\bowtie)$

Max Planck Institute for Polymer Research, Ackermannweg 10,

55128 Mainz, Germany

e-mail: musyanovych@mpip-mainz.mpg.de
}

\author{
A. Ziegler $\cdot$ K. Landfester $\cdot$ A. Musyanovych \\ Institute of Organic Chemistry III-Macromolecular Chemistry \\ and Organic Materials, University of Ulm, \\ Albert-Einstein-Allee 11, \\ 89081 Ulm, Germany
}

\section{Introduction}

Poly(vinylphosphonic acid; PVPA) and its copolymers found increasing interest in many fields of application. Generally, they are used as a model system for the study of protonconducting mechanism of phosphonated polymers in fuel cells [1-3]; vinylphosphonic acid-based hydrogels show improved proliferation and cell adhesion, which have been successfully used in the field of tissue engineering [4, 5]. Further applications of vinylphosphonic acid-containing copolymers are related to the conductive blends [6], separation membrane materials [7], and dental cements [8]. The synthesis of functional polymers and hydrogels based on vinylphosphonic acid via bulk (co)polymerization was described recently by Bingöl et al. [9]. Komber et al. studied the polymerization of PVPA and the corresponding methyl ester by nuclear magnetic resonance (NMR [10]. Various phosphonated methacrylate copolymer particles prepared by seeded semicontinuous emulsion polymerization were synthesized in the past [11]. Due to the high-interaction area, nanoparticles are widely used as adhesives or solid supports for biomolecules. The high affinity of phosphonate groups to different metal ions like $\mathrm{Cu}(\mathrm{II}), \mathrm{Co}(\mathrm{II}), \mathrm{Ag}(\mathrm{I}), \mathrm{Ni}(\mathrm{II})$, and $\mathrm{Cr}$ (III) can be controlled by the $\mathrm{pH}$ value, which leads to different applications such as dispersing agents [12]. Moreover, phosphonate and phosphate groups show excellent adsorption behavior onto metal oxide surfaces [13-17]. Therefore, such phosphonated particles can be used, for example, for the modification of aluminum oxide surfaces for corrosion protection or for artificial surfaces like titanium implants for biomedical coatings. Aqueous dispersions containing copolymers with phosphate groups were synthesized in emulsion polymerization and applied as coatings for corrosion protection [18]. Adler et al. [19, 20] reported the synthesis of different phosphate functionalized poly 
(butyl acrylate-co-styrene) microgels by a two-step emulsion polymerization process. The obtained microgels were brought onto the aluminum panels. Performed industrial tests revealed that the adsorbed nanoparticles have outstanding corrosion inhibition effects.

By the miniemulsion technique, well-defined nanoparticles with controlled particle size can be achieved in the presence of low surfactant amounts, which is beneficial, if the resulting particles are going to be used for coatings or biomedical applications. The principle of miniemulsion is based on the creation of narrowly sized monomer droplets by applying high shear, e.g., ultrasonication. In the subsequent polymerization process, the monomer droplets are converted into polymer particles without changing their identity [21]. Recently, carboxyl- and amino-functionalized polystyrene latex particles with different surface group densities were successfully synthesized by the miniemulsion copolymerization of styrene and acrylic acid or 2-aminoethyl methacrylate hydrochloride $[22,23]$. The effect of the comonomer amount and the surfactant type on the particle sizes and size distribution was determined by dynamic light scattering (DLS) and transmission electron microscopy (TEM). The presence of a fluorescent dye within a polymeric matrix made it possible to use the resulting particles, for example, as cell markers. It was shown that the cell uptake of the particles increases with increasing surface charges $[24,25]$. To the best of our knowledge, the synthesis of vinylphosphonic acid (VPA)based styrene or methyl methacrylate (MMA) copolymer nanoparticles by polymerization in miniemulsions was not described in the literature before. Here, we report the synthesis and characterization of the fluorescent surface phosphonate-functionalized polymer nanoparticles. The synthesis of phosphonated polymer nanoparticles is performed in a miniemulsion system by free-radical copolymerization of vinyl phosphonic acid and styrene or MMA. It will be shown that it is possible to control the particle size by variation of the surfactant amount, and the density of phosphonate groups at the surface could be adjusted by the used amount of VPA. Furthermore, the kinetic behavior of the VPA copolymerization with styrene or MMA studied by reaction calorimetry and monomer conversion rate is discussed in detail.

\section{Experimental part}

\section{Materials}

Styrene (Merck) and methyl methacrylate (Merck) were freshly distilled under reduced pressure before use. Vinylphosphonic acid was washed with diethyl ether and dried in vacuum to remove an inhibitor. All the other reagents and solvents were commercial products and were used without further purification: the hydrophobe, i.e., hexadecane (Aldrich, 99\%) and the hydrophobic initiator 2,2'-azobis(2-methylbutyronitrile; V59) from Wako Chemicals, Japan, the surfactants Lutensol AT50 from BASF, which is a poly(ethylene oxide)-hexadecyl ether with an EO block length of about 50 units, and sodium dodecyl sulfate (SDS) purchased from Aldrich. The fluorescent dye $N$-(2,6-diisopropylphenyl)perylene3,4-dicarbonacidimid (PMI; Fig. 1) was a gift from BASF. During the experiments, demineralized water was used.

Synthesis of phosphonate-functionalized polymer nanoparticles by miniemulsion process

The surface phosphonate-functionalized polymer particles were synthesized by free-radical copolymerization in miniemulsion polymerization. In Table 1, the amounts of the reagents are shown. For the synthesis of polySt-polyVPA latex particles, a given amount of styrene and vinyl phosphonic acid, $250 \mathrm{mg}$ of hexadecane, $3 \mathrm{mg}$ of PMI, and $100 \mathrm{mg}$ of initiator V59 were added as dispersed phase into $24 \mathrm{~g}$ of water containing $200 \mathrm{or} 400 \mathrm{mg}$ of the nonionic surfactant, Lutensol AT50 (or $72 \mathrm{mg}$ of the ionic surfactant SDS). After $1 \mathrm{~h}$ of stirring at the highest speed level (approximately 1,000 rpm), the mixture was homogenized by ultrasonication for $120 \mathrm{~s}$ at $90 \%$ intensity (Branson sonifier W450 Digital, one half-inch tip) at $0{ }^{\circ} \mathrm{C}$ in order to prevent polymerization of the monomer(s). Polymerization was carried out at $72{ }^{\circ} \mathrm{C}$ under stirring overnight. The preparation of the polyMMA-polyVPS nanoparticles was accomplished by the same procedure.

\section{Analytical methods}

The average particle sizes and the polydispersity index $(P D I)$ were determined by DLS using a Zeta Nanosizer (Malvern Instruments, UK) at a temperature of $20^{\circ} \mathrm{C}$ and a single scattering angle of $173^{\circ}$. DLS measurements give a value called $\mathrm{Z}$-average size (or cumulant mean), which is an intensity mean, and the PDI. The solid content of the sample was measured gravimetrically. All samples which were polymerized employing the nonionic surfactant Lutensol AT50 were cleaned by repetitive centrifugation/ redispersion in demineralized water. The latexes synthesized with ionic surfactant, i.e., SDS, were purified by

Fig. $1 N$-(2,6-diisopropylphenyl) perylene-3,4-dicarbonacidimid (PMI)

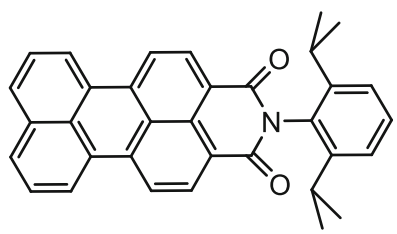


Table 1 Ingredients for the polymerization of phosphonate-functionalized polymer particles by the miniemulsion technique

\begin{tabular}{ll}
\hline Ingredients & Amount $(\mathrm{g})$ \\
\hline Styrene or MMA & $6.0-5.4$ \\
Vinyl phosphonic acid & $0.0-0.6(0-10$ wt $\%$ of VPA based on total styrene or MMA amount $)$ \\
Oil-soluble initiator V59 & 0.1 \\
Hydrophobe hexadecane & 0.25 \\
Fluorescent dye PMI & 0.003 \\
Non-ionic surfactant Lutensol AT50 & 0.2 or 0.4 \\
Ionic surfactant SDS & 0.072 \\
Demineralized water & 24.0 \\
\hline
\end{tabular}

dialysis using the Millipore membrane (MWCO 100000). The density of phosphonate groups on the particle surfaces was estimated by the titration against the opposite positively charged polyelectrolyte poly(diallyldimethyl ammonium chloride; PDADMAC) using a particle charge detector (Mütek GmbH, Germany) in combination with a Titrino Automatic Titrator (Metrohm AG, Switzerland). For the measurement, $10 \mathrm{~mL}$ of the latex sample with a solid content of $1 \mathrm{~g} / \mathrm{L}$ was used. The amount of groups per gram of polymer was calculated from the consumed volume of polyelectrolyte $[17,18]$. The zeta potentials were measured in $10^{-3} \mathrm{M}$ of potassium chloride solution with the Zeta Nanosizer (Malvern Instruments, UK) at $20^{\circ} \mathrm{C}$. The amount of fluorescent dye PMI in milligrams per gram of the freezedried polymer was determined using UV/VIS-spectroscopy (UV/VIS spectrometer Lambda 16, Perkin-Elmer). Dissolved in $2.5 \mathrm{~g}$ of THF was $7.8 \mathrm{mg}$ of the dried latex sample. The absorbance at the peak maximum in the spectra at the wavelength of $480 \mathrm{~nm}$ was analyzed. The morphology of the particles was examined by transmission electron microscopy (Philips EM400) at an acceleration voltage of $80 \mathrm{kV}$. For the TEM imaging, the latexes were diluted in demineralized water, and a drop of the solution was placed on a carbon-coated copper grid. The samples were dried overnight at room temperature. Additional contrasting was not applied. The calorimetric measurements were carried out in a reaction calorimeter (Micro Reaction Calorimeter, Thermal Hazard Technology). A given amount of monomer, VPA, initiator, fluorescent dye, and hexadecane, as described above, was dispersed in the continuous phase containing $200 \mathrm{mg}$ of Lutensol AT50 in $24 \mathrm{~g}$ of demineralized water. After $1 \mathrm{~h}$ of stirring at the highest speed level, the mixture was homogenized by ultrasonication at $0^{\circ} \mathrm{C}(120 \mathrm{~s}$ at $90 \%$ intensity). Afterwards, a calorimeter tube was filled in with $0.5 \mathrm{~mL}$ of the miniemulsion and placed into the calorimeter aluminum block containing the Peltier elements. The polymerization was carried out inside a calorimeter tube equipped with a magnetic stirring bar at $72^{\circ} \mathrm{C}$. The measurements were stopped when the heat flow was back at constant values of approximately 0 . The theoretical reaction heat for styrene homopolymerization is $\Delta H_{\mathrm{R}}=$ $-73 \mathrm{~kJ} / \mathrm{mol}$ and $\Delta H_{\mathrm{R}}=-58 \mathrm{~kJ} / \mathrm{mol}$ for MMA [26]. The monomer conversion, as a function of reaction time, was determined by integration of the reaction heat curves using the Origin program.

\section{Results and discussion}

Phosphonate-functionalized polystyrene nanoparticles with different surfactant types

To determine the influence of the surfactant on the particle size and size distribution, polySt-polyVPA copolymer particles were synthesized in the presence of the ionic, i.e., SDS or nonionic, i.e., Lutensol AT 50, respectively. The synthesis of the particles was carried out with various amounts of vinylphosphonic acid ( $1 \mathrm{wt} \%$ to $10 \mathrm{wt} \%$ related to the total amount of monomers) at $72{ }^{\circ} \mathrm{C}$ in miniemulsion process. The polymerization was started by using the oilsoluble hydrophobic initiator V59. In this case, the formation of water soluble oligomers in the continuous phase could be minimized. In order to suppress the Ostwald ripening of the droplets (i.e., the monomer diffusion from small droplets to larger ones through the continuous phase), the hydrophobe hexadecane was used. The particle sizes and size distribution of different vinylphosphonic acid-containing polystyrene particles synthesized with SDS or Lutensol AT50 were determined by DLS, and the obtained results are summarized in Table 2.

As expected, the VPA-containing polystyrene copolymer particles stabilized with an even smaller amount of ionic surfactant $(1.2 \mathrm{wt} \%$ SDS related to the total amount of monomers) are smaller in size as compared with the particles obtained with the nonionic one at 5.5 times higher surfactant concentration. The electrostatic repulsion originated from the SDS molecules is stronger than the steric one from the poly(ethylene oxide) chains of the nonionic 
Table 2 Particle size distribution of polySt-polyVPA particles synthesized in the presence of ionic or nonionic surfactant

\begin{tabular}{|c|c|c|c|c|c|c|c|c|}
\hline \multirow[t]{2}{*}{ VPA amount, wt $\%{ }^{\mathrm{a}}$} & \multirow[t]{2}{*}{ Sample name } & \multicolumn{3}{|c|}{ Synthesized with $1.2 \mathrm{wt} \% \mathrm{SDS}^{\mathrm{a}}$} & \multirow[t]{2}{*}{ Sample name } & \multicolumn{3}{|c|}{ Synthesized with $6.6 \mathrm{wt} \%$ Lutensol AT50 } \\
\hline & & $D_{\mathrm{Z}},(\mathrm{nm})^{\mathrm{b}}$ & $P D I$ & $\begin{array}{l}\text { Solid content, } \\
(\mathrm{wt} \%)^{\mathrm{c}, \mathrm{d}}\end{array}$ & & $D_{\mathrm{Z}},(\mathrm{nm})^{\mathrm{b}}$ & $P D I$ & $\begin{array}{l}\text { Solid content, } \\
(\mathrm{wt} \%)^{\mathrm{c}, \mathrm{e}}\end{array}$ \\
\hline 0 & AZ-50a-0V-SDS & 90 & 0.02 & 18.7 & AZC-MES-0 & 176 & 0.04 & 20.8 \\
\hline 1 & AZ-50b-1V-SDS & 102 & 0.02 & 19.5 & AZC-MES-1 & 184 & 0.04 & 20.1 \\
\hline 3 & AZ-50c-3V-SDS & 104 & 0.01 & 20.2 & AZC-MES-3 & 151 & 0.06 & 21.1 \\
\hline 5 & AZ-50d-5V-SDS & 105 & 0.01 & 18.5 & AZC-MES-5 & 149 & 0.02 & 19.7 \\
\hline 10 & AZ-50e-10V-SDS & 112 & 0.03 & 15.8 & AZC-MES10 & 145 & 0.12 & 20.7 \\
\hline
\end{tabular}

${ }^{\mathrm{a}}$ Related to the total amount of monomers, ${ }^{\mathrm{b}}$ determined by DLS, ${ }^{\mathrm{c}}$ determined gravimetrically, ${ }^{\mathrm{d}}$ theoretical solid content corresponds to $21.1 \%$, e theoretical solid content corresponds to $21.9 \%$

Lutensol AT50. Furthermore, due to the low molecular weight of SDS, larger a surface area can be stabilized, resulting in the formation of smaller-size droplets and subsequent final polymer particles. This difference is in evidence by comparing the nonfunctionalized polystyrene particles AZ-50a0V-SDS and AZC-MES-0, i.e., the polystyrene particles synthesized with SDS are only being half the size of the Lutensol AT50-stabilized latexes. In general, the average size of the particles synthesized with SDS and Lutensol AT50 is in a range of 90 to $110 \mathrm{~nm}$ and 140 to $180 \mathrm{~nm}$, respectively. Moreover, it can be seen from Table 2 that the size of the particles obtained with SDS continuously increases with the increasing VPA amount. The same trend was observed in the case of carboxyl-functionalized polystyrene latex particles obtained by copolymerization of styrene and acrylic acid in the presence of SDS [22]. The increase in diameter is a result of a growing outer layer, which is rich in hydrophilic comonomer (either acrylic acid [22] or VPA) units.

The hydrodynamic size of polySt-polyVPA latex particles stabilized with Lutensol AT50 first increases (with $1 \mathrm{wt} \% \mathrm{VPA}$ ) and then decreases with the increase in the VPA amount. VPA is a hydrophilic monomer possessing two $p K_{\mathrm{a}}$ values (2.6 and 7.3), and, therefore, during the polymerization, it mainly stays in the continuous phase. However, due to the high number of the droplets and, thus, an interaction of the surface area, we can assume that a part of the phosphonate groups is arranging at the monomer droplet/water interface. Indeed, the surface tension measurements revealed that VPA can serve as a low molecular weight surfactant $(\gamma \approx 60 \mathrm{mN} / \mathrm{m})$. The amount of $1 \mathrm{wt} \%$ VPA introduced for copolymerization is not sufficient to influence the stabilizing properties of Lutensol AT50. However, with increasing amount of phosphonate groups, the number of charges rises. This leads to the additional electrostatic stabilization of the droplets and subsequent smaller particle sizes.

The values of the $P D I$ are lower than 0.1 , indicating the size monodispersity of the latexes. Only the particles synthesized with $10 \mathrm{wt} \%$ VPA and stabilized with Lutensol AT50 seem to have some polydispersity in size $(P D I=0.12)$. However, we should bear in mind that at a high ratio of hydrophilic comonomer (e.g., VPA) the resulting particles possess a "core-shell" morphology. The outer layer mainly consists of the hydrophilic comonomer present in the form of noncompact polymeric chains. Due to their mobility, the average hydrodynamic size of the particles could be affected, resulting in a broadening of the size distribution.

\section{Copolymerization of VPA with different monomer types}

In comparison to the polySt-polyVPA nanoparticles stabilized with Lutensol AT50, PMMA-based particles were synthesized using the same polymerization procedure. Table 3 summarizes the characterization data of polySt-polyVPA and polyMMA-polyVPA nanoparticles obtained with various VPA amounts. The Lutensol AT50 content was kept constant at $6.6 \mathrm{wt} \%$ related to the monomer phase.

The phosphonated PMMA particles are obtained in a size range from 150 to $230 \mathrm{~nm}$ with $P D I$ values lower than 0.1 . In the case of polySt-polyVPA particles, the increase in introduced VPA amount results first in an increase of the particle size and then a decrease. This trend was observed for both types of particles (Table 3) with only one difference: that polyMMA-polyVPA particles are bigger in size compared with the polystyrene ones. This can be explained by difference in the hydrophilicity of styrene and MMA. The water solubility of styrene and MMA corresponds to $0.24 \mathrm{~g} / \mathrm{L}$ and $15.9 \mathrm{~g} / \mathrm{L}$, respectively. On the one hand, it can be assumed that, due to a higher water solubility of MMA, a part of the monomer might be polymerized in the continuous phase via homogeneous nucleation. The formed polymeric (or oligomeric) chains precipitate onto the particle surface, increasing, therefore, the size of the final particles. In the case of styrene polymerization, the polymeric chains are nucleated mainly inside the droplets. On the other hand, the amount of used 
Table 3 Characteristics of polystyrene- and PMMA-based latex particles as a function of VPA amount. All samples were stabilized with $6.6 \mathrm{wt} \%$ Lutensol AT50 related to the total amounts of monomers

\begin{tabular}{|c|c|c|c|c|c|c|c|c|}
\hline \multicolumn{2}{|l|}{ Sample name } & \multirow[t]{2}{*}{ Amount of VPA wt $\%{ }^{a}$} & \multicolumn{2}{|c|}{$D_{\mathrm{Z}}^{\mathrm{b}}(\mathrm{nm})$} & \multicolumn{2}{|l|}{$P D I$} & \multicolumn{2}{|c|}{ Solid content ${ }^{\mathrm{c}, \mathrm{d}}(\%)$} \\
\hline PS & PMMA & & PS & PMMA & PS & PMMA & PS & PMMA \\
\hline AZC-MES-0 & AZ45a-0VPA & 0 & 176 & 182 & 0.04 & 0.09 & 20.8 & 20.7 \\
\hline AZC-MES-1 & AZ45b-1VPA & 1 & 184 & 229 & 0.04 & 0.10 & 20.1 & 16.8 \\
\hline AZC-MES-3 & AZ45c-3VPA & 3 & 151 & 201 & 0.06 & 0.07 & 21.1 & 15.4 \\
\hline AZC-MES-5 & AZ45d-5VPA & 5 & 149 & 205 & 0.02 & 0.09 & 19.7 & 15.2 \\
\hline AZC-MES10 & AZ45e-10VPA & 10 & 145 & 150 & 0.12 & 0.04 & 20.7 & 14.8 \\
\hline
\end{tabular}

${ }^{\mathrm{a}}$ Related to the total amounts of monomers, ${ }^{\mathrm{b}}$ determined by DLS, ${ }^{\mathrm{c}}$ determined gravimetrically, ${ }^{\mathrm{d}}$ theoretical solid content corresponds to $21.9 \%$

hexadecane (which was kept constant for styrene and MMA polymerization) is not sufficient to suppress the Oswald ripening of MMA-based droplets, thus resulting in their growth and subsequent bigger particles. Monomer diffusion through the continuous phase also has an effect on the solid content of the final latexes. More coagulum and, therefore, latexes with a lower solid content were obtained in the case of phosphonated PMMA particles.

Polymerization with lower amount of Lutensol AT50

Because of the additional stabilization by the charge originated from phosphonate groups, latex particles were synthesized using half of the surfactant Lutensol AT50 amount (i.e., $3.3 \mathrm{wt} \%$ Lutensol AT50 instead of $6.6 \mathrm{wt} \%$ related to the total amount of monomers). To get comparable values, the amount of VPA, styrene, and MMA were kept the same as for polymerization conducted with $6.6 \mathrm{wt} \%$ Lutensol AT50. In Table 4, the particle size, size distribution, and solid content for polySt-polyVPA and polyMMA-polyVPA copolymer particles are summarized.

From the obtained data, it could be seen that the solid content of the particles synthesized with $3.3 \mathrm{wt} \%$ of Lutensol AT50 is lower in comparison with those synthe- sized with $6.6 \mathrm{wt} \%$ of surfactant, indicating a higher tendency for coagulum formation. In particular, polyStpolyVPA and polyMMA-polyVPA latexes with $1 \mathrm{wt} \% \mathrm{VPA}$ were obtained with nearly half of a solid content as compared with theoretical value $(21.4 \%)$. As it was mentioned before, the amount of $1 \mathrm{wt} \%$ VPA is not sufficient to improve the stability of the droplets. At the same time, bridging polymerization of VPA could be additionally responsible for the system destabilization.

The average size of polystyrene- or PMMA-based particles obtained with $3.3 \mathrm{wt} \%$ Lutensol AT50 based on total amount of monomer is bigger as in the case of using $6.6 \mathrm{wt} \%$ of surfactant. Phosphonated polystyrene-based particles are in the size range of 210 to $250 \mathrm{~nm}$, whereas the size of polyMMA-polyVPA particles varies between 210 and $310 \mathrm{~nm}$. It is interesting to note that, for both monomers (styrene or MMA), with the addition of VPA, the particle size first increases and then continuously decreases. The same trend was also observed by using $6.6 \mathrm{wt} \%$ Lutensol AT50, thus indicating that the particle formation mechanism under studied conditions does not depend on the surfactant amount. Figure 2 shows the plots of average particle diameters as a function of VPA and Lutensol AT50 amounts.
Table 4 Characteristics of polystyrene- and PMMA-based latex particles as a function of VPA amount. All samples were stabilized with $3.3 \mathrm{wt} \%{ }^{\mathrm{a}}$ Lutensol AT50
${ }^{a}$ Related to the total amount of monomers, ${ }^{\mathrm{b}}$ determined by DLS,

${ }^{c}$ determined gravimetrically,

$\mathrm{d}$ theoretical solid content corresponds to $21.4 \%$

\begin{tabular}{|c|c|c|c|c|c|c|c|c|}
\hline \multicolumn{2}{|c|}{ Sample name } & \multirow[t]{2}{*}{ Amount of VPA wt $\%^{\mathrm{a}}$} & \multicolumn{2}{|c|}{$D_{\mathrm{Z}}^{\mathrm{b}}[\mathrm{nm}]$} & \multicolumn{2}{|l|}{$P D I$} & \multicolumn{2}{|c|}{ Solid content ${ }^{\mathrm{c}, \mathrm{d}}(\%)$} \\
\hline PS & PMMA & & PS & PMMA & PS & PMMA & PS & PMMA \\
\hline $\begin{array}{l}\text { AZ-31a } \\
0 \text { VPA }\end{array}$ & $\begin{array}{l}\text { AZ-42a } \\
0 \text { VPA }\end{array}$ & 0 & 208 & 301 & 0.03 & 0.22 & 19.0 & 17.9 \\
\hline $\begin{array}{l}\text { AZ-31b } \\
1 \text { VPA }\end{array}$ & $\begin{array}{l}\text { AZ-42b } \\
1 \text { VPA }\end{array}$ & 1 & 247 & 312 & 0.03 & 0.14 & 15.7 & 13.3 \\
\hline $\begin{array}{l}\text { AZ-31c } \\
3 \text { VPA }\end{array}$ & $\begin{array}{l}\text { AZ-42c } \\
3 \text { VPA }\end{array}$ & 3 & 231 & 250 & 0.05 & 0.07 & 19.2 & 14.9 \\
\hline $\begin{array}{l}\text { AZ-31d } \\
5 \text { VPA }\end{array}$ & $\begin{array}{l}\text { AZ-42d } \\
5 \text { VPA }\end{array}$ & 5 & 228 & 220 & 0.06 & 0.03 & 16.3 & 17.4 \\
\hline $\begin{array}{l}\text { AZ-31e } \\
10 \mathrm{VPA}\end{array}$ & $\begin{array}{l}\text { AZ-42e } \\
\text { 10VPA }\end{array}$ & 10 & 210 & 207 & 0.09 & 0.12 & 17.7 & 18.2 \\
\hline
\end{tabular}




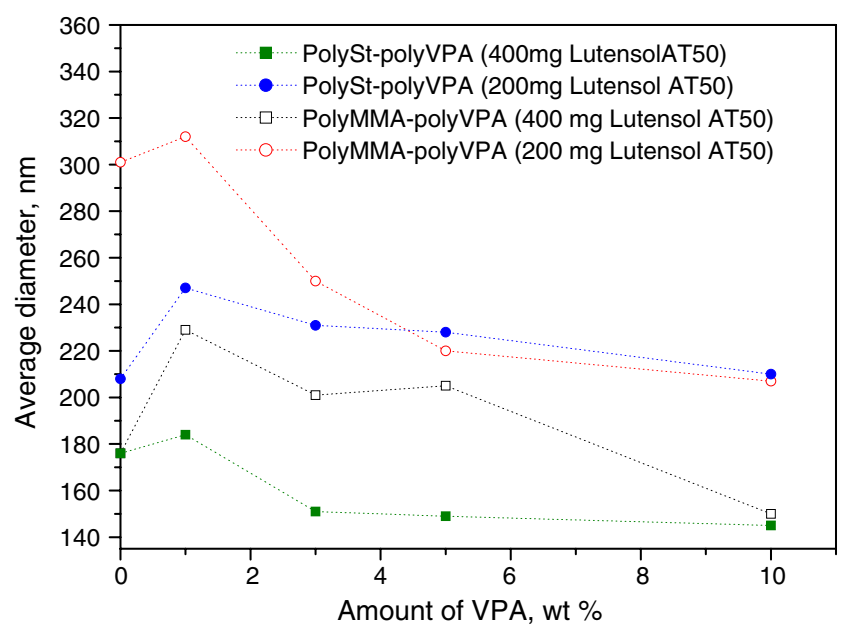

Fig. 2 Average particle size of polySt-polyVPA (filled square and filled circle) and polyMMA-polyVPA (open square and open circle) with different amounts of Lutensol AT50

\section{Transmission Electron Microscopy}

Morphology, average size, and size distribution of the obtained polymer particles were studied by TEM. In the case of polySt-polyVPA particles synthesized with $10 \mathrm{wt} \%$ VPA (sample AZC-MES10), the DLS values $(P D I=0.12)$ show some size polydispersity, which we refer to the mobility of the VPA-rich chains on the particle surface. The TEM measurements support our hypothesis. As it can be seen from Fig. 3b, the particles possess monomodal size distribution. In general, for both copolymers with different VPA amounts a, hexagonal arrangement of the particles can be clearly seen in the TEM images (Figs. 3 and 4), indicating not only the size uniformity but also their spherical shape. In contrast to the polystyrene-based particles, PMMA particles are not thermally stable against the heat of the electron beam under TEM measurement conditions. Therefore, the melting of PMMA-based particles mainly at the edges could be observable at the images (Fig. 4). The values of particle size measured by DLS were supported by different TEM images of the samples.

Although the usage of $3.3 \mathrm{wt} \%$ Lutensol AT50 results in some coagulum formation, the individual particles were found to be monodisperse in size. Thus, the use of a low surfactant amount is sufficient enough to get copolymer particles of VPA with either styrene or MMA in a controlled way, i.e., with the controlled size and size distribution.

\section{Surface group titration}

The electrokinetic properties of the latex particles were described in terms of zeta potential. At $\mathrm{pH} 10$ the nonfunctionalized polymer particles show minor negative charges -3 to $-5 \mathrm{mV}$, which, according to the literature, corresponds to the hydroxyl ion adsorption at the particle/ water interface [18]. For phosphonated latex particles, with the increase of VPA amount, the zeta potential decreases. This dependency is a further evidence for the arrangement of the charged hydrophilic phosphonate groups at the particle surface. The zeta potential values for polyStpolyVPA and polyMMA-polyVPA particles were in the range between -12 to $20 \mathrm{mV}$ and -20 to $42 \mathrm{mV}$ at $\mathrm{pH} 10$, respectively. From the obtained data, it can be observed that the amount of phosphonate groups is higher on the surface of PMMA-based particles. In order to quantify the density of functional groups on the particle surface, titration against an opposite-charged polyelectrolyte was performed $[22,27-29]$. The titration was based on the interaction between the charged phosphonate groups at the particle surface and the chains of opposite-charged polyelectrolyte
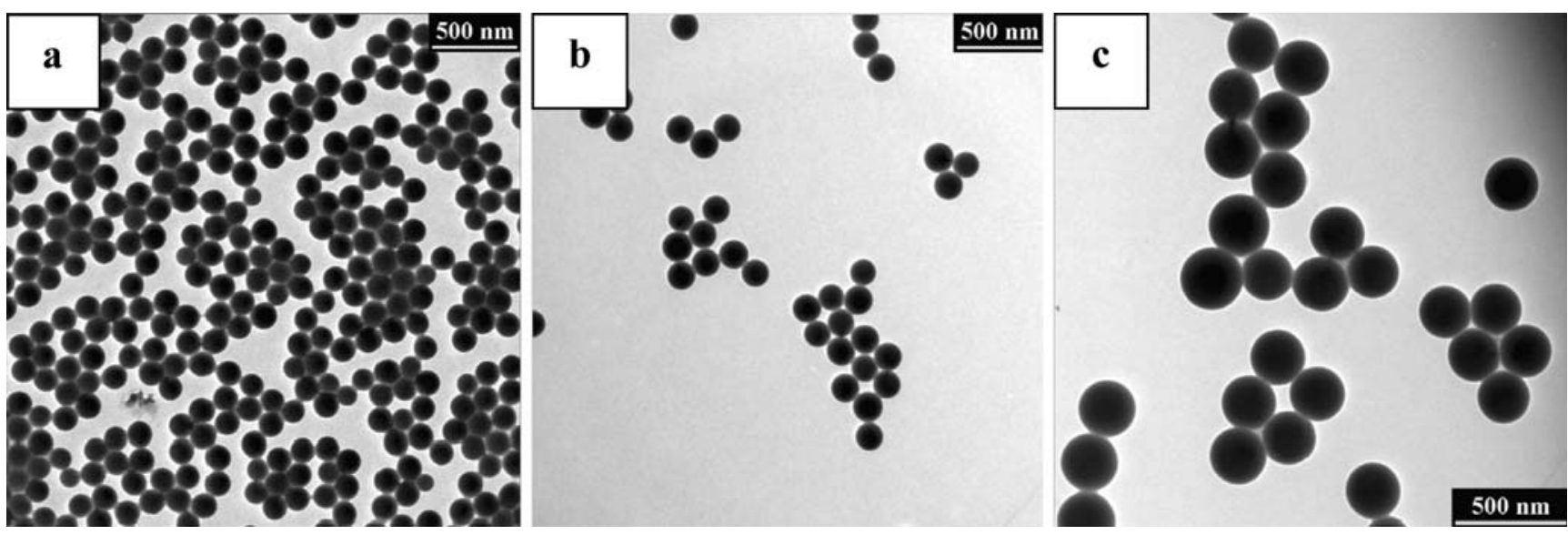

Fig. 3 TEM images of the a pure polystyrene (AZC-MES-0) and the polySt-polyVPA particles with $10 \mathrm{wt} \%$ VPA stabilized with b $6.6 \mathrm{wt} \%$ Lutensol AT50 (AZC-MES10) and c $3.3 \mathrm{wt} \%$ Lutensol AT50 (AZ-15e10VPA). Scale bar corresponds to $500 \mathrm{~nm}$ 

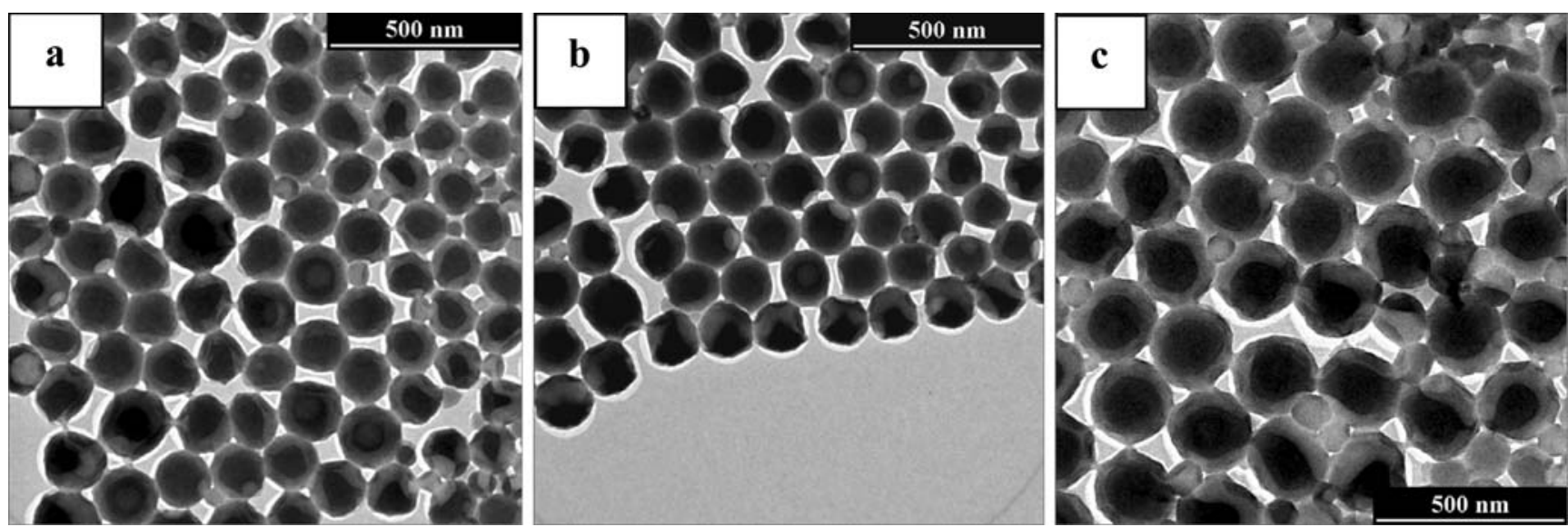

Fig. 4 TEM images of a pure PMMA polymer particles (sample AZ45a0VPA) and the polyMMA-polyVPA particles with 10 wt $\%$ VPA stabilized with b $6.6 \mathrm{wt} \%$ Lutensol AT50 (AZ45e-10VPA) and c $3.3 \mathrm{wt} \%$ Lutensol AT50 (AZ-42e10VPA). Scale bar corresponds to $500 \mathrm{~nm}$

polyDADMAC with low molecular weight. The measurements were carried out at $\mathrm{pH} 10$. At this high $\mathrm{pH}$ value, all phosphonate groups are deprotonated in aqueous solution $\left(p K_{1}=2.6\right.$ and $p K_{2}=7.3$ ). The amount of functional groups can be calculated from the values of solid content, size of the polymer particles, and the polymer density using the equations given in the experimental part. The surface group density for polySt-polyVPA and polyMMA-polyVPA particles synthesized at two different concentrations of Lutenol AT50 is summarized in Table 5. From the obtained results, it can be seen that, although the amount of phosphonate groups per particle are higher in the case of using $3.3 \mathrm{wt} \%$ Lutensol AT50 (due to the bigger particle size), a significant difference in the groups' density (per $\mathrm{nm}^{2}$ ) was not observed. Comparing both types of polymeric particles, the titration results show that the density of surface phosphonate groups increases with the increase in the VPA concentration used during the copolymerization. The samples with $10 \mathrm{wt} \%$ VPA lead to nearly the double amount of phosphonate groups as compared with the samples with $5 \mathrm{wt} \%$ of VPA. Furthermore, the amount of functional groups is much higher in the case of polyMMApolyVPA particles than for polySt-polyVPA ones. These data support the zeta potential measurements, which show more negative charges for PMMA-based particles.

\section{Determination of the PMI amount}

Due to the presence of the fluorescent dye, which is encapsulated inside the polymeric matrix, it is possible to

Table 5 Amount of the functional groups at the particle surfaces stabilized with different amounts of Lutensol AT50

\begin{tabular}{|c|c|c|c|c|c|c|}
\hline \multirow{2}{*}{\multicolumn{2}{|c|}{ Sample name }} & \multirow[t]{3}{*}{ Amount of VPA $\left(w t^{0}{ }^{a}\right)$} & \multirow{2}{*}{\multicolumn{2}{|c|}{$\frac{\text { PolySt-PolyVPA }}{\text { Amount of groups per }}$}} & \multicolumn{2}{|c|}{ PolyMMA-PolyVPA } \\
\hline & & & & & & \\
\hline PolySt-PolyVPA & PolyMMA-PolyVPA & & particle & $\mathrm{nm}^{2}$ & particle & $\mathrm{nm}^{2}$ \\
\hline \multicolumn{7}{|c|}{ Synthesized with $6.6 \mathrm{wt} \%$ Lutensol AT50 $0^{\mathrm{a}}$} \\
\hline AZC-MES-0 & AZ45a-0VPA & 0 & 0 & 0 & 0 & 0 \\
\hline AZC-MES-1 & AZ45b-1VPA & 1 & 14883 & 0.14 & 39520 & 0.24 \\
\hline AZC-MES-3 & AZ45c-3VPA & 3 & 14319 & 0.20 & 34251 & 0.27 \\
\hline AZC-MES-5 & AZ45d-5VPA & 5 & 19520 & 0.28 & 44865 & 0.34 \\
\hline AZC-MES10 & AZ45e-10VPA & 10 & 27067 & 0.41 & 46630 & 0.66 \\
\hline \multicolumn{7}{|c|}{ Synthesized with 3.3 wt $\%$ Lutensol AT50 ${ }^{\mathrm{a}}$} \\
\hline AZ-31a-0VPA & AZ-42a-0VPA & 0 & 0 & 0 & 0 & 0 \\
\hline AZ-31b-1VPA & AZ-42b-1VPA & 1 & 19157 & 0.10 & 76415 & 0.25 \\
\hline AZ-31c-3VPA & AZ-42c-3VPA & 3 & 31920 & 0.19 & 54950 & 0.28 \\
\hline AZ-31d-5VPA & AZ-42d-5VPA & 5 & 40807 & 0.25 & 48632 & 0.32 \\
\hline AZ-31e-10VPA & AZ-42e-10VPA & 10 & 58160 & 0.42 & 84764 & 0.63 \\
\hline
\end{tabular}

${ }^{\mathrm{a}}$ Related to the total amount of monomers 
apply the obtained particles in various biomedical tests, for example, as cell markers [24, 25]. The amount of incorporated fluorescent dye PMI was measured by UV spectroscopy. A calibration curve of PMI in THF was recorded, and the linear equation of this standard was used for calculations. Table 6 summarizes the PMI amounts for the polySt-polyVPA and polyMMA-polyVPA particles synthesized with different concentrations of Lutensol AT50.

The maximum encapsulated PMI amount corresponds to the introduced amount of PMI, i.e., $0.5 \mathrm{mg}$ PMI per $1 \mathrm{~g}$ of the polymer. From the results presented in Table 6 , it can be seen that the PMI encapsulation yields are between 62 and $98 \mathrm{wt} \%$.

Kinetic behavior of the copolymerization in miniemulsion

The kinetic behavior of free-radical copolymerization of VPA with styrene or MMA in the presence of the hydrophobic initiator V59 in miniemulsion was studied by calorimetry. The calorimetric studies focused on the influence of VPA fraction on the kinetic behavior and reaction time of the copolymerization with styrene or MMA. For heterophase polymerization, three nucleation mechanisms are discussed: droplet nucleation, homogenous nucleation, and micellar nucleation [30-33]. For miniemulsion polymerization, two different nucleation mechanisms are possible. The first and preferred mechanism is the droplet nucleation where the polymerization is directly started by radicals entering the monomer droplets and the reaction with the monomer there explains the possibility of using oil-soluble initiators in miniemulsion process [34].
The second possible mechanism is the homogeneous nucleation; however, it is discussed to be of minor importance for the polymerization of styrene in miniemulsion [8] and is always intended to be minimized. For classical emulsion polymerization, the intervals of the reaction mechanism were described by Harkins [35]. The first interval is called the nucleation interval (interval I); when under the usage of hydrophilic initiator, the formation of free primary radicals takes place in the water phase. The radicals enter micelles, and the second interval (interval II) starts with the complete adsorption of the surfactant on the surface of the growing latex particles. The monomer diffuses from the monomer droplets, which serve as reservoirs and react with the growing latex particles. The reaction rate is constant in interval II. The monomer is consumed in the third interval (interval III), and the reaction speed decreases exponentially. In the fourth interval (interval IV), a gel effect (Trommsdorff-Norish effect) can be observed due to the high viscosity of monomer-swollen polymer particles. In contrast to emulsion polymerization, in a miniemulsion system, three distinguished intervals are categorized for the kinetic curves. The first one is the nucleation interval which is quite shorter as in emulsion polymerization. The interval III with constant average number of radicals and interval IV (a gel effect) are present, but the second interval of constant reaction rate is missing in miniemulsion polymerization $[30,35]$. The kinetic behavior of VPA and styrene or MMA copolymerization performed in miniemulsion was studied using $3.3 \mathrm{wt} \%$ Lutensol AT50 and hydrophobic initiator V59. The calorimetric curves for polystyrene- and PMMA-based particles are presented in Fig. 5.

Table 6 Amount of encapsulated PMI in polySt-polyVPA and polyMMA-polyVPA particles obtained with different concentrations of Lutensol AT50

\begin{tabular}{|c|c|c|c|c|}
\hline \multirow[t]{2}{*}{ VPA amount, $\left(w t^{0}{ }^{a}\right)$} & \multicolumn{2}{|l|}{ PolySt-polyVPA } & \multicolumn{2}{|c|}{ PolyMMA-polyVPA } \\
\hline & Sample name & PMI amount $\left(\mathrm{mg} / \mathrm{g}_{\mathrm{Pol}}\right)$ & Sample name & PMI amount $\left(\mathrm{mg} / \mathrm{g}_{\mathrm{Pol}}\right)$ \\
\hline \multicolumn{5}{|c|}{ Synthesized with 6.6 wt $\%$ Lutensol AT50 $0^{\mathrm{a}}$} \\
\hline 0 & AZC-MES-0 & 0.37 & AZ45a-0VPA & 0.43 \\
\hline 1 & AZC-MES-1 & 0.49 & AZ45b-1VPA & 0.49 \\
\hline 3 & AZC-MES-3 & 0.39 & AZ45c-3VPA & 0.31 \\
\hline 5 & AZC-MES-5 & 0.33 & AZ45d-5VPA & 0.39 \\
\hline 10 & AZC-MES10 & 0.46 & AZ45e-10VPA & 0.36 \\
\hline \multicolumn{5}{|c|}{ Synthesized with 3.3 wt $\%$ Lutensol AT50 ${ }^{\mathrm{a}}$} \\
\hline 0 & $\mathrm{AZ}-31 \mathrm{a} 0 \mathrm{VPA}$ & 0.45 & $\mathrm{AZ}-42 \mathrm{a} 0 \mathrm{VPA}$ & 0.42 \\
\hline 1 & AZ-31b1 VPA & 0.49 & AZ-42b1 VPA & 0.48 \\
\hline 3 & AZ-31c3 VPA & 0.41 & AZ-42c3 VPA & 0.37 \\
\hline 5 & AZ-31d5 VPA & 0.35 & AZ-42d5 VPA & 0.45 \\
\hline 10 & AZ-31e10VPA & 0.39 & AZ-42e10VPA & 0.46 \\
\hline
\end{tabular}

${ }^{a}$ Related to the total amount of monomers 
From Fig. 5, it can be seen that the heat flow time curves have different shapes and reaction times for styrene- and MMA-based (co)polymerizations. The obtained heat of reaction data of homopolymerization is in good agreement with the theoretical values. The theoretical heat of styrene and MMA homopolymerization corresponds to $\Delta H_{\mathrm{R}}=-73 \mathrm{~kJ} / \mathrm{mol}$ and $\Delta H_{\mathrm{R}}=-58 \mathrm{~kJ} / \mathrm{mol}$, respectively [26]. The difference between styrene homopolymerization and VPA-containing samples are clearly seen in the presence of a double peak and shorter reaction time. A possible reason for this could be the big difference in water solubility of VPA and styrene.

After placing of the miniemulsion sample in the calorimeter block, a nonisothermal reaction period was detected (not included in Fig. 5). For both styrene- and PMMA-based particles, the real time of polymerization start corresponds to approximately $300 \mathrm{~s}$. Typical three intervals for polymerization in miniemulsion can be observed in polystyrene-based systems. The first interval is very short. After approximately 1,250 s (21 min) till 6,250 s (104 min), the exponential decrease of the curves is observed; this is due
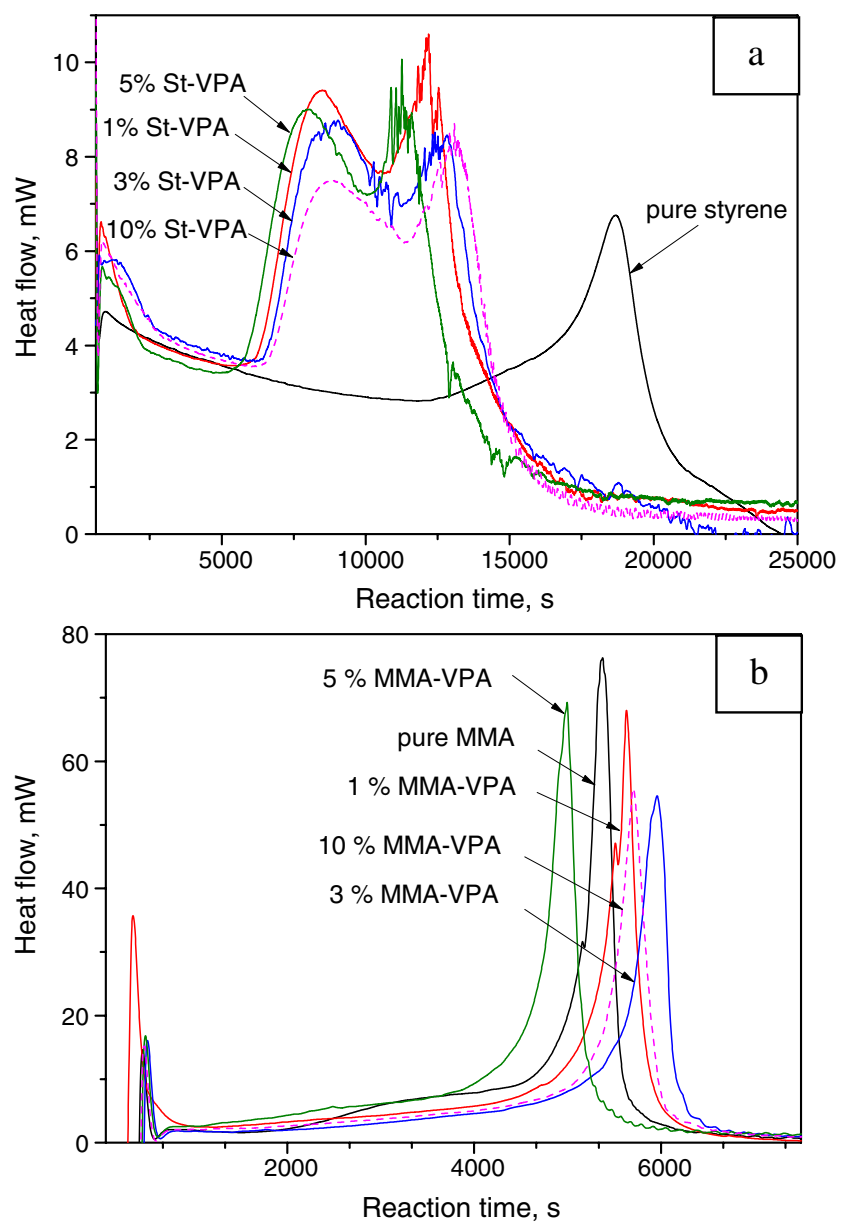

Fig. 5 Calorimetric curves of VPA copolymerization with a styrene and b MMA to the consumption of the monomer inside the droplets (interval III). For the VPA-containing samples, the gel peak is obtained between $10,000 \mathrm{~s}(167 \mathrm{~min})$ and $15,000 \mathrm{~s}$ (250 $\mathrm{min}$ ), and for styrene homopolymerization, approximately after 19,000 s (317 $\mathrm{min})$. In contrast to polystyrene, the heat flow curves for MMA-based samples have only one peak that corresponds to the gel effect.

From the literature, it is known that the reaction rate can be influenced by different parameters such as particle size, surface functionalization of the particles, and water solubility of the monomers. In general, the reaction time for styrene-based polymerizations is longer as compared with MMA-based ones. This is in agreement with the propagation rate constant of both monomers, i.e., $k_{\mathrm{p}}$ corresponds to $426 \mathrm{l} / \mathrm{mol} \mathrm{s}$ and $1041 \mathrm{l} / \mathrm{mol} \mathrm{s}$ for styrene and MMA, respectively. It could be assumed that, in the case of MMAbased (co)polymerization, an overlap of two different nucleation processes (droplet and homogeneous) is taking place due to the high water solubility of MMA and VPA. Furthermore, according to Norrish and Smith [36], the gel
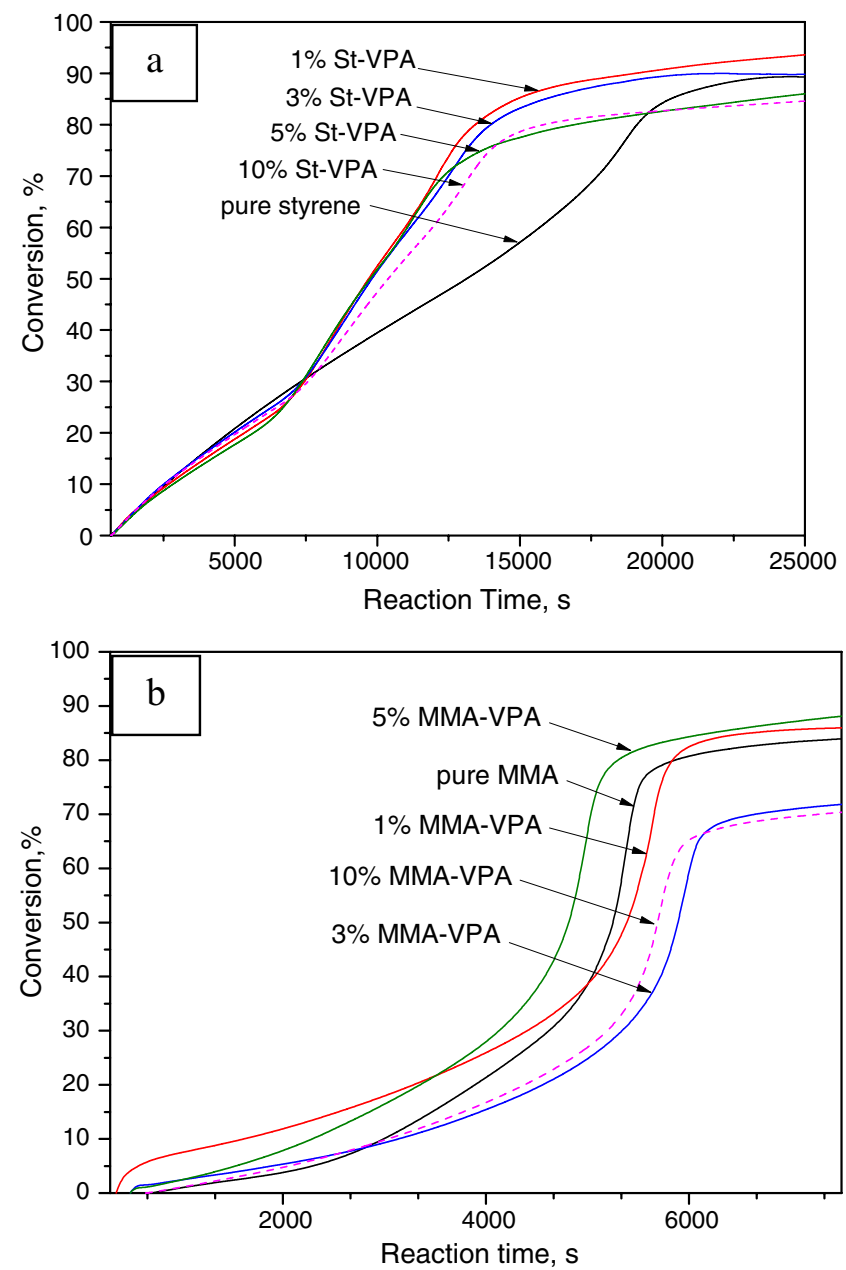

Fig. 6 Monomer conversion versus reaction time of VPA copolymerization with a styrene and b MMA 
effect in MMA polymerization is more pronounced as compared with that in the styrene polymerization.

The monomer conversion curves of polystyrene- and PMMA-based particles obtained from the integration of calorimetric data (Fig. 5) are plotted in Fig. 6 as a function of the reaction time.

The reference $\Delta H_{\mathrm{R}}$ values for styrene or MMA copolymerization with VPA are not available in the literature. Therefore, for all systems, the reference $\Delta H_{\mathrm{R}}$ values for styrene or MMA homopolymerization were taken as the reference points for complete conversion. Although this might lead to some deviation in the obtained results, the data presented in Fig. 6 could be compared qualitatively. Moreover, the monomer conversion as a function of reaction time was determined independently using the gravimetric analysis. The shape of the curves and the values in the end of polymerization were similar to those presented in Fig. 6.

For pure styrene 1 and $3 \mathrm{wt} \% \mathrm{VPA}$, the monomer conversion is around $90-95 \%$. For the samples with higher VPA amount (5 and $10 \mathrm{wt} \%$ ), the monomer conversion is about $85 \%$ which can be an effect of hydrophilic monomer presence. Till $6,000 \mathrm{~s}$ (100 $\mathrm{min}$ ) of reaction time and $30 \%$ of conversion, the curves show the same slope, which is a part of interval III of the miniemulsion kinetic. Afterwards, the conversion increases rapidly in the case of copolymerization and reaches plateau after approximately $15,000 \mathrm{~s}$ (250 $\mathrm{min})$. For styrene homopolymerization, the plateau is reached after approximately 20,000 s. The conversion values for PMMAbased samples are lower in comparison with styrene ones (between $73 \%$ and $92 \%$ ), and no data correlation was observed as a function of VPA amount.

\section{Conclusions}

In the present paper, we report the synthesis of phosphonated polystyrene and PMMA nanoparticles based on vinylphosphonic acid by free-radical copolymerization performed in miniemulsion. Depending on the amount and type of the surfactant used (ionic or nonionic), phosphonatefunctionalized particles in a size range from 102 to $312 \mathrm{~nm}$ can be obtained. The surface groups' density can be adjusted by the concentration of the VPA comonomer. The amount of phosphonate groups increases with the increase in the VPA fraction used during the copolymerization. Moreover, the functionalization of the particles is much more effective in the case of using MMA as a basis monomer than polystyrene. From the calorimetric data, it could be observed that only styrene-based (co)polymerization followed typical miniemulsion polymerization mechanism. The heat flow time curves of copolymerization of styrene with VPA differ from the styrene homopolymerization, which is due to the difference in water solubility of the monomers. We assumed that, in the case of MMA-based (co)polymerization, an overlap of two different nucleation processes (droplet and homogeneous) is taking place due to the high water solubility of MMA and VPA.

Acknowledgments This work was supported by Landesstiftung Baden-Württemberg. The authors would like to thank Mr. Reinhard Weih (University of Ulm) for his technical assistance with TEM measurement and Mr. Christian Wohnhaas for the support in experimental work.

Open Access This article is distributed under the terms of the Creative Commons Attribution Noncommercial License which permits any noncommercial use, distribution, and reproduction in any medium, provided the original author(s) and source are credited.

\section{References}

1. Lee YJ, Bingöl B, Murakhtina T, Sebastiani D, Meyer WH, Wegner G, Spiess HW (2007) J Phys Chem B 111:9711

2. Steiniger H, Schuster M, Kreuer KD, Kaltbeitzel A, Bingöl B, Meyer WH, Schauff S, Brunklaus G, Maier J, Spiess HW (2007) J Phys Chem 9:1764

3. Kaltbeitzel A, Schauff S, Steininger H, Bingöl B, Brunklaus G, Meyer WH, Spiess HW (2007) Solid State Ionics 178:469

4. Tan J, Gemeinhart RA, Ma M, Saltzmann WM (2005) Biomaterials 26:3663

5. Gemeinhart RA, Bare CM, Haasch RT, Gemeinhart EJ (2006) Biomed Mater Res A 78A:433

6. Sahoo SK, Nagarajan R, Roy S, Samuelson LA, Kumar J, Cholli AL (2004) Macromolecules 37:4130

7. Park CH, Nam SY, Lee YM (1999) J Appl Polym Sci 74:83

8. Jiang DD, Yao Q, Mc Kinney MA, Wilkie CA (1999) Polym Degrad Stab 63:423

9. Bingöl B, Strandberg C, Szabo A, Wegner G (2008) Macromolecules 41:2785

10. Komber H, Steinert V, Voit B (2008) Macromolecules 41:2119

11. Gaboyard M, Jeanmaire T, Pichot C, Hervaud Y, Boutevin B (2003) J Polym Sci Part A 41:2469

12. Rivas BL, Pereira E (2003) Macromol Symp 193:237

13. Liu HB, Venkataraman NV, Spencer ND, Textor M, Xiao SJ (2008) Chem Phys Chem 9:1979

14. Textor M, Ruiz L, Hofer R, Rossi A, Feldman K, Haehner G, Spencer ND (2000) Langmuir 16:3257

15. Jaehne E, Oberoi S, Adler HJP (2008) Prog Org Coatings 61:211

16. Gnauck M, Jaehne E, Blaettler T, Tosatti S, Textor M, Adler HJP (2007) Langmuir 23:377

17. Pawsey S, Yach K, Reven L (2002) Langmuir 18:5205

18. Schmidt-Hansberg T, Adler HJP, Henke A, Patent DE Nr. 19911 $842 \mathrm{~A} 1$

19. Henke A, Jaehne E, Adler HJP (2001) Macromol Symp 164:1

20. Adler HJP, Jaehne E, Henke A, Yan L, Pich A (2002) Macromol Symp 187:53

21. Landfester K (2003) Top Curr Chem 227:75

22. Musyanovych A, Rossmanith R, Tontsch C, Landfester K (2007) Langmuir 23:5367

23. Holzapfel V, Musyanovych A, Landfester K, Lorenz MR, Mailänder V (2005) Macromol Chem Phys 206:2440

24. Jain A, Jain SK (2008) Eur J Pharm Sci 35:404 
25. Kim BS, Kim CS, Lee KM (2008) Arch Pharm Res 31:1050

26. Brandrup J, Immergut EH (1989) Wiley Intersci Polymer Handbook

27. Boeckenhoff K, Fischer WR (2001) Anal Bioanal Chem 371 (5):670

28. Munoz-Espi R, Qi Y, Lieberwirth I, Gomez CM, Wegner G (2006) Chem Eur J 12(1):118

29. Tauer K (1998) Macromolecules 31(26):9390

30. Bechthold N, Landfester K (2000) Macromolecules 33:4682
31. El-Aasser MS, Miller CM (1997) Polymeric dispersions principle and applications, JA. Asua Ed. Dordrecht p109

32. Samer CJ, Schork FJ (1997) Polym React Eng 5:85

33. Choi YT, Sudol ED, Vanderhoff JW, El-Aasser MS (1985) J Polym Sci 23:2973

34. Blythe PJ, Klein A, Phillips JA, Sudol ED, El-Aasser MS (1999) J Polym Sci Part A 37:4449

35. Harkins WD (1947) J Am Chem Soc 69:1428

36. Norrish RGW, Smith RR (1942) Nature 150:336 